\title{
Retroalimentación en lecciones de Estatística con Recursos Tecnológicos
}

\author{
Carolina Carvalho \\ cfcarvalho@ie.ulisboa.pt \\ Instituto de Educação da \\ Universidade de Lisboa, Portugal
}

\author{
Maria Niedja Martins \\ marianiedjamartins@campus.ul.pt \\ Instituto de Educação da \\ Universidade de Lisboa, Portugal
}

\author{
Carlos Monteiro \\ cefmonteiro@gmail.com \\ Universidade Federal de \\ Pernambuco, Brasil.
}

Recibido: Setiembre 5, 2016

Aceptado: Marzo 10, 2017

Resumen. En muchos cursos la retroalimentación del profesor es oral, como cuando interactúa con los alumnos, o escrita, como cuando evalúa sus producciones escritas. Pero los recursos tecnológicos y el software educativo están creando nuevos contextos de aprendizaje. En esta comunicación los objetivos son (a) retomar ideas sobre la retroalimentación en situaciones de aprendizaje de contenidos de Estadística, mediadas por el uso de recursos tecnológicos; (B) reflejar cómo estos recursos generan potencial en la participación con las tareas; (C) revelar la necesidad de que los profesores tengan la oportunidad de trabajar con estos recursos antes de usarlos con los alumnos. Los resultados ilustran que cuando los profesores tienen la oportunidad de explorar contextos donde se recurre a estas herramientas, ellos también desarrollan conocimientos y competencias que les dan confianza para implementarlos en sus clases.

Palabras clave: Lecciones de Estadística, Clases de Estatística, Retroalimentación del profesor, Recursos tecnológi$\cos$

\begin{abstract}
In many classrooms, the teacher's feedback is oral, when assessing with the students or written, when assess their papers. However, technological resources and educational software are creating new learning contexts. The aims of this communication are (a) refresh ideas about feedback in situations of learning of Statistical contents mediated by the use of technology; (b) reflect how these resources generate potential in the engagement with tasks; (c) reveal the need for teachers to have the opportunity to work with these resources before using them with students. The results illustrate that when teachers have the opportunity to explore contexts where these tools are used they also develop knowledge and skills that give them confidence to implement them in their classroom.
\end{abstract}




\section{Introducción}

En los últimos años, una de las modificaciones más profundas en la enseñanza de la estadística ha sido consecuencia de la tecnología (Jolliffe [15], 2007). De hecho, vemos a un significativo número de recursos tecnológicos que se pueden utilizar en las lecciones cuando se trabaja con este contenido disciplinario. Las calculadoras, computadoras y software educativo provocaron, junto a una creciente facilidad de acceso a datos reales en Internet, una revolución en muchas aulas. En diversas situaciones de enseñanza de la estadística se recurre a investigaciones estadísticas con datos reales, utilizando contextos ricos y significativos de aprendizaje (Garfield \& Ben-Zvi [11], 2010). Los avances en la tecnología y los múltiples recursos tecnológicos, hoy disponibles, son incorporados en la educación estadística de modo diversificado, en particular como instrumento de apoyo a los alumnos (y a los propios profesores) durante la exploración y análisis de datos reales, en la resolución dinámica de actividades estadísticas o en la comprensión de conceptos e ideas estadísticas ya que promueven el desarrollo del razonamiento estadístico de los alumnos (Ben-Zvi \& Garfield [4], 2004).

Según Ben-Zvi [2] (2000), son varios los atributos de las computadoras que parecen contribuir al desarrollo del sentido y de los significados en los alumnos, en particular: la capacidad de operar de forma rápida y precisa, para enlazar dinámicamente varias representaciones, simplificar los procedimientos, proporcionar retroalimentación y transformar una representación como un todo en un objeto manipulable. Es decir, "implican una reorganización de la actividad cognitiva y un cambio del foco de atención hacia un nivel cognitivo superior"(p 141).

Sin embargo, el acceso a recursos tecnológicos no garantiza, por sí solo, un aprendizaje efectivo, es fundamental el papel activo del profesor en la institución educativa y el soporte del desarrollo del razonamiento de los alumnos, enfocándose tanto en las representaciones gráficas construidas, como en las cuestiones que se explotan para asegurar que ellos desarrollen la comprensión de la articulación necesaria entre estos aspectos (Henriques \& Antunes [14], 2014). Es por eso que la retroalimentación del profesor, que ocurre después de un comportamiento, un desempeño o una actitud, consistente en la información recibida del esfuerzo desarrollado para alcanzar un determinado objetivo y concretar una determinada tarea (Wiggins [29], 2012) Una situación más dinámica como cuando el alumno trabaja como software, también necesita ser renovado.

En un contexto de aula, y pensando en el profesor, la retroalimentación es una consecuencia de la actuación de un alumno y su finalidad es proporcionar información relacionada con la tarea o proceso de aprendizaje, cuyo objetivo es mejorar el desempeño en una tarea específica y/o el entendimiento de un determinado asunto (Sadler [27], 1989). Esta retroalimentación puede influir en mucho como los estudiantes aprenden ciertos contenidos, pero la literatura todavía no es consensuada sobre lo que se considera como una retroalimentación eficaz (Bergh, Ros \& Beijaard [5], 2013).

\subsection{Retroalimentación y recursos tecnológicos}

Ainley y Monteiro [1](2008) al analizar contenidos de currículos de Estadística en Brasil y en el Reino Unido y su implementación enfatizan un cierto abismo entre lo que se planea y lo que se concreta en los primeros años de escolaridad, considerando que a pesar de que los responsables de la elaboración 
de los currículos valoran la participación activa de los estudiantes en la construcción de los conocimientos, no hay una especificación clara en los documentos de cómo los profesores podrían desarrollar tal enfoque. Los autores van más lejos cuando se refieren a que estos objetivos, al formularse de forma vaga, se convierten en grandes desafíos para los profesores de los primeros años, que pueden ellos mismos tener un conocimiento poco profundo sobre ideas estadísticas y, por lo tanto, de materiales de apoyo más detallados (por ejemplo libros didácticos, orientaciones curriculares, ejemplos de actividades, criterios de evaluación). En los dos contextos nacionales mencionados, la interpretación de los objetivos curriculares en esos materiales de apoyo se aparta de las orientaciones más desafiantes de resolución de problemas y realización de investigaciones, por lo que aún es necesario contar con referencias en la formación de los profesores para que ellos sean conscientes de su papel en el proceso de aprendizaje de los alumnos (Quintas, Tomás Ferreira \& Oliveira [24], 2013). Y más aún cuando se recurre a tecnologías para crear ambientes de aprendizaje más atractivos para los alumnos.

De hecho, como alertan Pratt, Davies y Conner [23] (2011) hace mucho que se apunta a la necesidad de modificar la formación de profesores, respecto a la educación estadística, atendiendo las dificultades de los profesores de lidiar con los conocimientos estadísticos y como trabajarlos recurriendo a la tecnología, ya que durante su formación rara vez se sumergen en situaciones similares a las que más tarde deben promover junto a sus alumnos.

En concreto, el software TinkerPlots tiene como principal objetivo favorecer las exploraciones de datos estadísticos y no presenta funciones que ofrezcan una retroalimentación automática a los usuarios sobre si los análisis y las representaciones de los datos son correctos o no. Por lo tanto, corresponde al profesor actuar (o dar retroalimentación) para que el alumno perciba si las resoluciones encontradas son adecuadas.

De acuerdo con Hattie [13] (2009), la retroalimentación busca reducir las discrepancias entre la comprensión y el desempeño actuales y una intención u objetivo de aprendizaje. En otras palabras, debe hacer que el estudiante pueda ir más lejos en sus desempeños y razonamientos.

Aunque el término retroalimentación forma parte del discurso de los profesores y está presente en muchas situaciones de su práctica lectiva, la literatura lo refiere como complejo y no siempre utilizado de forma eficaz por el profesor (Fonseca et al. [9], 2015). Varios autores han considerado la retroalimentación con tres aristas: cognitiva, motivacional y afectiva. Por ejemplo, Brookhart [7] (2008) describe la retroalimentación eficaz en términos de dos aristas: la cognitiva y la motivacional. La arista cognitiva tiene que ver con el suministro de información necesaria a los alumnos para poder comprender dónde se encuentran en su aprendizaje y qué tienen que hacer para mejorar los resultados. La arista motivacional se refiere al desarrollo en los alumnos de la "sensación de que tienen control sobre su propio aprendizaje"(Brookhart [7], 2008, p.2).

Hay un consenso general en la literatura de que la retroalimentación debe darse a un nivel que los alumnos puedan comprender (Orsmond, Merry, \& Reiling [22], 2005), y será más eficaz si se proporciona en un ambiente de clases donde la respuesta, incluso cuando sea incorrecta, es valorada como una oportunidad de reflexión en lugar de ser ofrecido como un juicio de valor (Weaver [28], 2006). Ser eficaz es ser claro, tener un propósito, ser significativo, compatible con el conocimiento previo de los alumnos y proporcionarle conexiones lógicas que le lleven a concentrarse en cómo mejorar los resultados (Hattie [13], 2009). 
La arista afectiva de la retroalimentación se revela cuando la información proporcionada por el profesor se centra en en el alumno y no en el desempeño o la comprensión del mismo. Cuando esto sucede, los alumnos pueden aumentar el miedo al fracaso, minimizar su esfuerzo o evitar los riesgos que resultan de un abordaje de tareas desafiantes (Black \& William [6], 1998).

Grieshaber [12] (2010) argumenta que cuando los alumnos usan computadoras en el aula existen varias oportunidades de retroalimentación e interacción generadoras de participación en las tareas. Así, aquella autora resalta que en situaciones de enseñanza y de aprendizaje mediados por computadoras, la organización y gestión de los pequeños grupos también afecta a lo que ocurre entre los alumnos y la productividad del grupo. Al trabajar con estas herramientas se crea la posibilidad de activar conocimientos anteriores y ampliarlos, generándose una oportunidad de verificar un cambio conceptual entre conceptos anteriores incompletos o incorrectos y nuevas (re)configuraciones más robustas.

Para ello el profesor debe identificar los momentos críticos y proporcionar la retroalimentación que permita este cambio conceptual.

Las situaciones de enseñanza mediadas por recursos tecnológicos remiten a una concepción de la actividad cognitiva donde la mediación y el papel de artefactos y herramientas cognitivas están presentes. La mediación se refiere al hecho de que nuestra interacción con el mundo recurre a signos y artefactos, y el ordenador es un artefacto que potencia conocimientos y procesos de aprendizaje pero que hace más complejo el uso de la retroalimentación por parte de los profesores. Las actividades que recurren al ordenador en el aula tienden a fomentar la colaboración entre los alumnos, al externar verbalmente el trabajo cognitivo realizado individualmente. A continuación, presentaremos algunas consideraciones sobre la retroalimentación en situaciones de formación de profesores.

\subsection{Retroalimentación en situaciones de aprendizaje de estadística mediadas} por TinkerPlots: potencial para la formación de profesores

Con el fin de discutir la retroalimentación del profesor en situaciones de aula donde se utilizaron recursos tecnológicos, vamos a discutir episodios referentes a situaciones de aprendizaje de un estudio desarrollado en Brasil (Martins [18], 2014) que exploró el aprendizaje del concepto de muestra con profesores de los años iniciales de la Enseñanza Fundamental. El enfoque del estudio de Martins no era la retroalimentación del profesor, sino el proceso de aprendizaje de nociones y conceptos estadísticos por medio del software TinkerPlots (Konold \& Miller [16], 2005).

En Brasil, la investigación en torno al uso de TinkerPlots entre profesores y estudiantes de diferentes niveles de escolaridad ha revelado resultados positivos en términos de aprendizajes de conceptos y desarrollo de raciocinios estadísticos más complejos entre los sujetos (Monteiro, Carvalho \& Ainley [19], 2013, Monteiro, Martins \& Carvalho [21], 2015). Tal vez porque este software crea un ambiente dinámico, en el cual los estudiantes pueden organizar y explorar diferentes representaciones gráficas de datos recurriendo a sofisticadas y rápidas formas de procesar, que en una forma tradicional obligaba a

Retroalimentación en lecciones de Estatística con Recursos Tecnológicos . C. Carvalho et al

Derechos Reservados (C) 2017 Revista digital Matemática, Educación e Internet (http://tecdigital.tec.ac.cr/revistamatematica/) 
cálculos rutinarios y largos. Las posibilidades de producir una diversidad de representaciones ofrecen condiciones para el análisis de hipótesis en el proceso de interpretación de datos pero también acentúa la necesidad de que los alumnos y los profesores no desvaloricen la necesidad del pensamiento estadístico incluso cuando se recurre a la tecnología (Pratt, Davies \& Conner [23], 2011).

La profesora participante del estudio de Martins [18] (2014) tenía 30 años y experiencia de 5 años como profesora de los años iniciales de la Enseñanza Fundamental, habiendo concluido la carrera de bachillerato en Pedagogía hacía año y medio, aproximadamente. La profesora afirmó que utilizaba el ordenador diariamente, pero no conocía el software TinkerPlots, ni utilizaba ningún otro software educativo con los alumnos así como que no había realizado ningún aprendizaje formal sobre muestreo.

Después de una sesión de familiarización con el software (Raberdel \& Waern [25], 2003), se le presentaron las principales herramientas del TinkerPlots, la profesora analizó una situación en el software sobre un piscicultor que almacenó en un tanque una población de 625 peces, donde algunos habían sido genéticamente modificados. Para identificar qué grupo de peces presentaba una modificación mayor, el piscicultor debería retirar gradualmente los peces del tanque y analizarlos en función del tamaño. Los datos sobre los peces se presentaban en TinkerPlots, y a partir de la selección de casos, era posible visualizarlos por medio de una tabla y de la herramienta Plot, en la que era posible visualizar una representación gráfica de los casos La profesora debería hacer el papel del piscicultor e identificar el grupo de peces con un mayor modificación genética al sacar muestras crecientes de los peces.

Campos et al. [8] (2011) conciben que el razonamiento sobre el muestreo es un conocimiento necesario para la construcción de un raciocinio estadístico. Esto porque adquirir conocimientos estadísticos presupone, entre otras cosas, "entender la relación entre la muestra y la población, lo que puede ser inferido con base en una muestra y desconfiar de inferencias hechas a partir de pequeñas muestras" (Campos et al. (En el caso de las mujeres).

En la tarea propuesta en el estudio de Martins [18] (2014), sobre los peces normales y genéticamente alterados, está presente la noción de que las muestras más grandes son más seguras para realizar inferencias a la población, ya que al aumentar la muestra se visualizan mejor las particularidades de la población a la que pertenece. En otras palabras, las muestras más grandes pueden representar mejor la variabilidad de la población. En la visión de Lopes, la cuestión de la variabilidad es un concepto clave en la estadística.

El raciocinio estadístico, como centro del proceso, tiene la variabilidad de hacer relaciones sobre el
problema investigado, de elaborar la construcción y el análisis de los datos. La variabilidad presente
en los datos determina una forma de pensar que requiere una combinación de ideas, lo que nos remite
a una intersección entre los razonamientos combinatorios, probabilísticos y estadísticos (Lopes[17],
2012, p. 167).

Sin embargo, en personas que no pasaron por una enseñanza formal sobre la estadística, no cabe esperar que la idea de buscar una mejor representación de la variabilidad de la población en las muestras surja espontáneamente. Al contrario de lo que se puede imaginar, algunas personas que entran en contacto por primera vez con problemas sobre la representatividad de las muestras tienden a creer que: 1) las muestras son siempre representativas de la población, sin importar su tamaño o la forma en que fueron seleccionadas; $\mathrm{O} 2$ ) de manera general las muestras son poco confiables, ya que son sólo parte de una población (Rubin, Bruce \& Tenney[26], 1991). 
Una manera de hacer que los sujetos enfrenten estas intuiciones, según las Directrices para la evaluación y la instrucción en la educación de la sociedad (GAISE [10], 2005), consiste en tomar muestras y analizar la variación de una misma medida estadística y compararla con el parámetro de la población respectiva. Tal diseño de tarea podría llevarlos a percibir que las muestras aleatorias de una misma población pueden variar un poco y no ser necesariamente un retrato fiel de la población, pero aún así, sobre la base de una muestra representativa, se pueden establecer inferencias para la población.

Para Monteiro y Martins [20] (2016), el uso del software educativo, por medio de la simulación, puede ser un elemento interesante para explorar las ideas iniciales sobre Estadística y Probabilidad que las personas traen consigo y, progresivamente, confrontar tales intuiciones. Ben-Zvi, Bakker y Makar [3] (2015), a su vez aclaran que al retirar muestras de un simulador repetidas veces, un individuo puede llegar progresivamente a conclusiones más claras del por qué esa muestra es o no representativa al analizar las distribuciones, compararlas y hacer comparaciones entre la variabilidad de los datos y las estadísticas extraídas de cada muestra.

La estrategia de la investigadora se centró en presentar las muestras crecientes de peces en el TinkerPlots, verificar la confianza de la profesora sobre sus inferencias, sugerir o no la necesidad de aumentar las muestras y de utilizar herramientas del software que faciliten la interpretación de los datos. Este proceso sólo pudo ocurrir a partir del intercambio de retroalimentación entre la docente y la investigadora. Sólo así podrían ofrecerse sugerencias que ayudara a la profesora a avanzar en la tarea, buscando el objetivo final del aprendizaje.

Un ejemplo de ese proceso de intercambio ocurrió cuando la investigadora seleccionó al azar algunos peces en el TinkerPlots y pidió que la participante analizara su representatividad, según los extractos de la entrevista:

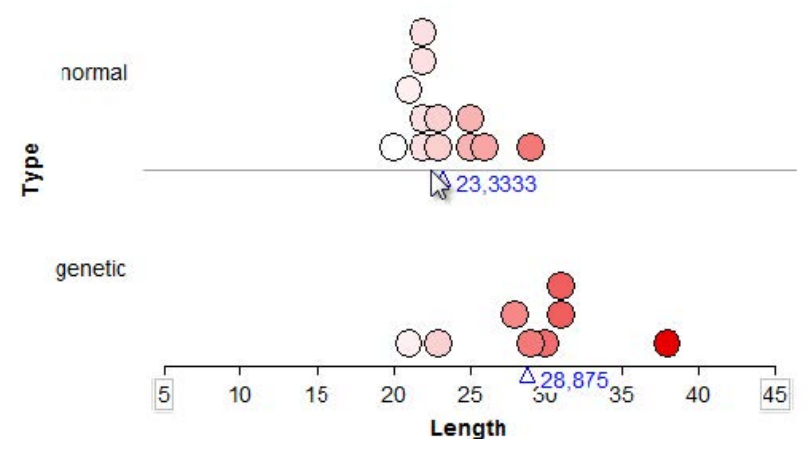

Figura 1.1: Pantalla de TinkerPlots 2.0 con una muestra de 10 peces.

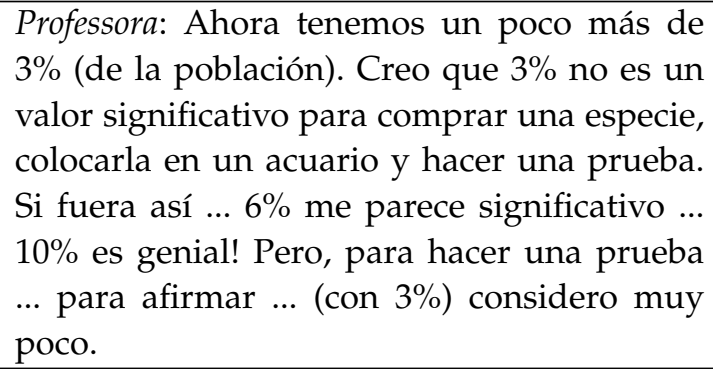
$3 \%$ (de la población). Creo que 3\% no es un valor significativo para comprar una especie, colocarla en un acuario y hacer una prueba. Si fuera así ... $6 \%$ me parece significativo ... $10 \%$ es genial! Pero, para hacer una prueba ... para afirmar ... (con $3 \%$ ) considero muy poco.

Conforme a la solicitud de la investigadora, la profesora analiza la representatividad de la muestra, indicando que la muestra no es significativa para realizar una inferencia a la población. 


\begin{tabular}{|l|l|}
\hline $\begin{array}{l}\text { Investigadora: (...) ¿Estás diciendo que tal vez } \\
\text { no sea significativo para el resto? }\end{array}$ & \\
\hline $\begin{array}{l}\text { Profesora: ¡Exacto! (...) Creo que si yo fuera } \\
\text { el piscicultor, no aceptaría esos datos. Hasta }\end{array}$ & $\begin{array}{l}\text { Con base en el cuestionamiento de la in- } \\
\text { vestigadora, la profesora explica de otra ma- } \\
\text { los peces genéticos se están mostrando? Es } \\
\text { porque no sabemos cuánto se tiene (total de } \\
\text { cada tipo de pez en la población). Si fuera } \\
\text { rencia con esa muestra. }\end{array}$ \\
$\begin{array}{l}50 \% \text { ahí podríamos considerarlo? Pero, ahí, } \\
\text { los peces fueron colocados allí? Puede que se } \\
\text { haya colocado sólo 10, sólo 5. }\end{array}$ & \\
\hline $\begin{array}{l}\text { Investigadora: Ok. Usted puede ver que se } \\
\text { han tomado } 12 \text { normales y } 8 \text { genéticamente } \\
\text { modificados. Como usted todavía no está } \\
\text { mostrando certeza en su respuesta, vamos a } \\
\text { tomar otros } 10 \text { peces, ok? }\end{array}$ & $\begin{array}{l}\text { En esa sección la investigadora identifica } \\
\text { que la respuesta de la profesora no pre- } \\
\text { sentó un nivel de confianza, ni una infe- } \\
\text { rencia. A partir de eso, la investigadora pro- } \\
\text { pone una alternativa para que la profesora } \\
\text { ofrezca esos elementos. }\end{array}$ \\
\hline
\end{tabular}

La interacción establecida entre la investigadora y la profesora se centra en la comprensión de la profesora sobre la representatividad de la muestra. Cuando la profesora admite no considerar la muestra suficientemente representativa de la población en función de su tamaño, la investigadora sugiere añadir más casos a la muestra. Esta estrategia resultó adecuada para aclarar la idea de que la muestra era suficiente para realizar una inferencia.

A partir del incremento de 10 casos en la muestra, conforme a la sugerencia de la investigadora, la profesora logró establecer un nivel de confianza elevado para la inferencia de los datos a la población, como muestra el diálogo:

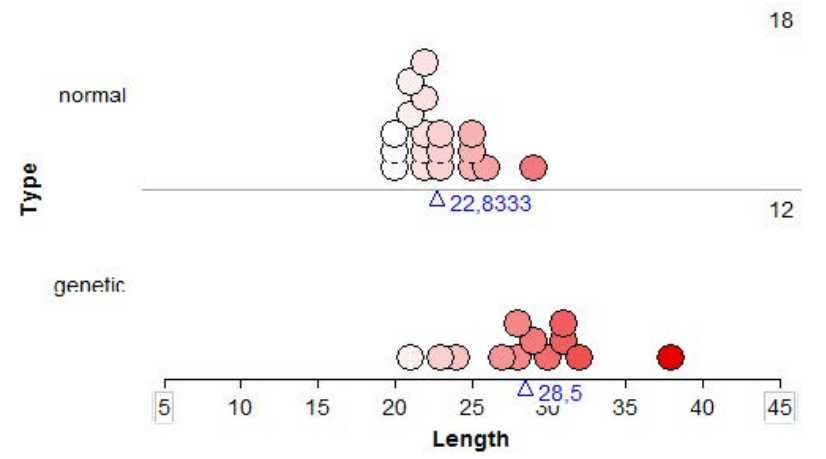

Figura 1.2: Pantalla de TinkerPlots 2. con una muestra de 30 peces. 


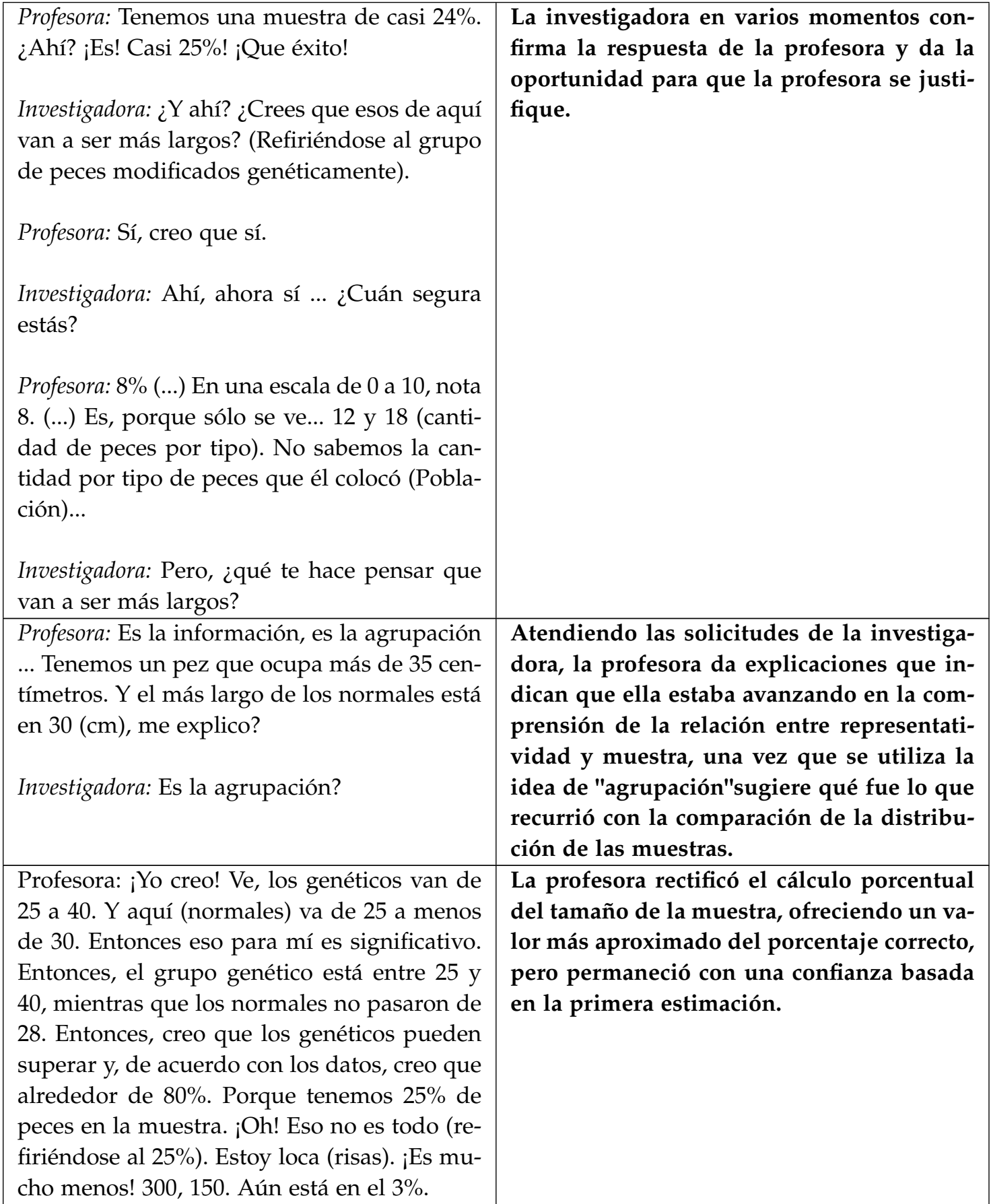

Es posible notar que el intento de establecer un nivel de confianza por la profesora relacionó el porcentaje del tamaño de la muestra con el tamaño de la población. Se observa que la profesora realiza un cálculo equivocado del porcentaje que representaba el tamaño de la muestra, pero no recibe una orientación de la investigadora para repensar su cálculo. Una intervención en ese momento podría haber sido eficaz si la investigadora estuviera cuestionando la relación establecida por la profesora entre 
el porcentaje del tamaño de la muestra y el nivel de confianza. Se percibe, que no hay aprovechado el error de la profesora, para que la misma reformule su nivel de confianza informal, tuvo consecuencias para el desarrollo de la tarea y de los niveles de confianza siguientes ofrecidos.

En algunos casos, la investigadora utilizó nuevamente la estrategia de aumentar la muestra, pero en todos esos momentos, la docente afirmó que la confianza sobre su certeza no aumentaba, como se muestra en los siguientes fragmentos:

\begin{tabular}{|c|c|}
\hline $\begin{array}{l}\text { Investigadora: Entonces, añadimos diez casos } \\
\text { más. Quedaron cuarenta casos ahí. Y usted } \\
\text { había dicho que tenía una certeza de } 8 \text {, es } \\
\text { así?! El promedio pasó de } 28 \text { a } 27,8 \text { y en los } \\
\text { normales se quedó } 23 \text {. Entonces, ¿crees que } \\
\text { ellos todavía van a ser más largos? ¿Tu con- } \\
\text { fianza ha aumentado o sigue igual? }\end{array}$ & $\begin{array}{l}\text { La investigadora centra su explicación en la } \\
\text { tarea de recapitular lo que la profesora hizo } \\
\text { hasta ese momento. }\end{array}$ \\
\hline $\begin{array}{l}\text { Profesora: (...) La confianza sigue siendo la } \\
\text { misma. }\end{array}$ & \\
\hline $\begin{array}{l}\text { Investigadora: Si en vez de tomar más diez } \\
\text { (peces), tomo el doble de los que se tienen } \\
\text { aquí? Entonces, voy a tomar cincuenta más y } \\
\text { va a quedar una muestra con } 100 \text { peces. ¿Y } \\
\text { ahora? }\end{array}$ & $\begin{array}{l}\text { La investigadora opta también por aumen- } \\
\text { tar al doble los casos, tratando de garantizar } \\
\text { una estrategia de aumento de las muestras, } \\
\text { de acuerdo a la Figura } 1.2 \text {. }\end{array}$ \\
\hline
\end{tabular}
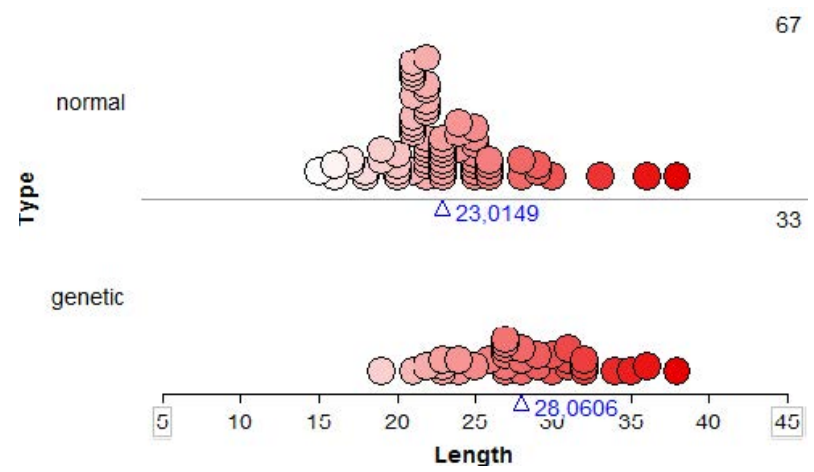

Figura 1.3: Pantalla de TinkerPlots 2.0 con una muestra de 100 peces. 


\begin{tabular}{|l|l|}
\hline $\begin{array}{l}\text { Profesora: Un momento... Ahora cuántos hay? } \\
\text { Un momento... } 600 \text { casos, } 300 \ldots 150 \ldots \text { Repite } \\
\text { la pregunta. }\end{array}$ & $\begin{array}{l}\text { La investigadora aclara el tamaño de la mu- } \\
\text { estra en relación con el tamaño de la pobla- } \\
\text { ción. }\end{array}$ \\
$\begin{array}{l}\text { Investigadora: He añadido el doble de los ca- } \\
\text { sos que habían. Entonces, ahora hay } 100 \text { ca- } \\
\text { sos en la muestra y con un tanque que tiene } \\
625 \text { (peces). Entonces, ahora, mirando hacia } \\
\text { aquí (gráfica), puedo afirmar con más cer- } \\
\text { teza o no, que los peces genéticamente modi- } \\
\text { ficados van a ser más largos? Para el tanque } \\
\text { todo, ¿no? }\end{array}$ & \\
Profesora: Sí! Si son más? Sí! & $\begin{array}{l}\text { Investigadora: La certeza que tenías antes era } \\
\text { de ocho, ahora aumentó o no? }\end{array}$ \\
$\begin{array}{l}\text { La estrategia de aumentar la muestra, uti- } \\
\text { lizada por la investigadora, parece no hacer } \\
\text { efecto en la comprensión de la profesora so- } \\
\text { bre la idea de que al aumentar la muestra } \\
\text { más representativa sería. }\end{array}$ \\
\hline
\end{tabular}

Como discutimos, el alto nivel de confianza ya atribuido por la profesora puede haber influido en permanecer con el mismo nivel de confianza. Sin embargo, si analizamos los intentos de retroalimentación en esta situación, percibimos que la investigadora continúa estableciendo la misma estrategia (aumentar la muestra) y las mismas preguntas. Así, una de las posibles causas, por las que la retroalimentación de la investigadora no ha funcionado, hace referencia a la claridad con que ella cuestionaba el nivel de confianza. La investigadora, entonces, reformuló su pregunta tomando en cuenta el nivel de confianza informal sobre la inferencia:

Investigadora: ¿Cuántos casos serían necesarios añadir en esa muestra para tener una certeza del $100 \%$ de que los genéticamente modificados realmente van a presentar una longitud mayor que los normales?

Profesora: Tendría que ver todos los casos.

Investigadora: Pero, ahí tienes 625. ¿Crees que sería posible que el piscicultor tome esa cantidad de peces tan rápidamente?

Profesora: Tomaría mucho tiempo. ¿Cuántos casos hemos tomado ya?
La investigadora realiza una retroalimentación a través de un nuevo cuestionamiento a la profesora. En vez de indicar simplemente la imposibilidad de realizar lo que profesora sugiere, la investigadora recuerda la situación problema del piscicultor relacionado con la tarea. 
Investigadora: 110.

Profesora: (Se queda pensando) Creo que 150.

La investigadora da un tiempo a la profesora para pensar y formular una respuesta.

Investigadora: Entonces tomaría 40 más, es así?

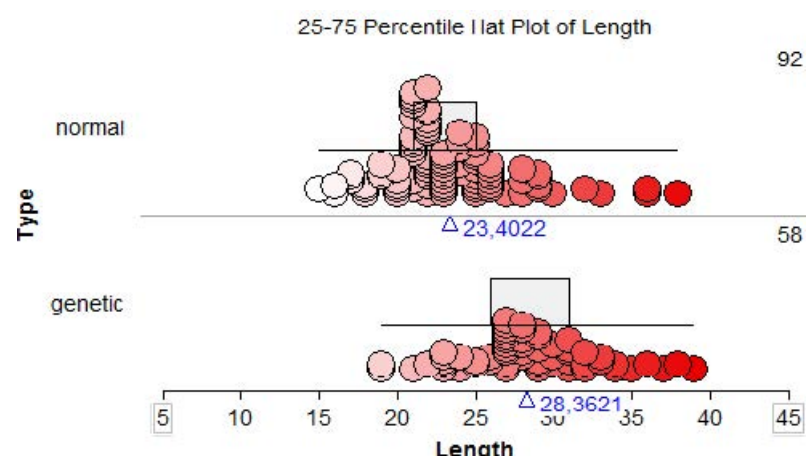

Figura 1.4: Pantalla de TinkerPlots 2.0 con una muestra de 150 peces.

\begin{tabular}{|l|l|}
\hline Profesora: ¿Acerté de lleno, vio? & $\begin{array}{l}\text { La reacción de la profesora denota involu- } \\
\text { cración en la tarea y el reconocimiento de la } \\
\text { respuesta correcta. }\end{array}$ \\
\hline Profestigadora: (risas). ¿Y ahí? & $\begin{array}{l}\text { La investigadora demuestra empatía expli- } \\
\text { citando la dimensión afectiva del diálogo. }\end{array}$ \\
\hline
\end{tabular}

El proceso establecido aquí ocurrió por medio de una constante negociación entre la investigadora y la docente, a fin de ajustar los cuestionamientos relacionados con la tarea a las impresiones que la profesora mantenía sobre los datos, con el objetivo de que la entrevistada obtuviera mayor confianza sobre sus respuestas.

El uso de ciertas herramientas también fue una estrategia adoptada por la investigadora y que pareció contribuir a que la profesora consolidara el análisis que hacía de las muestras. 


\begin{tabular}{l|l|}
\hline $\begin{array}{l}\text { Investigadora: ¿Usted percibió cuánto varió el } \\
\text { promedio? (...) ¿Cuánto varió el promedio? }\end{array}$ & $\begin{array}{l}\text { La investigadora plantea una situación para } \\
\text { estimular a la profesora a percibir que otros } \\
\text { aspectos fueron importantes para identifi- } \\
\text { car la representatividad de las muestras. Lo } \\
\text { hizo por medio de una pregunta directa so- } \\
\text { bre la variación de la media. }\end{array}$ \\
$\begin{array}{l}\text { cadosora: Fue } 27 \text { aquí (genéticamente modifi } 23 \text {... (normales). La media fue } \\
\text { constante y con pocas variaciones. }\end{array}$ & $\begin{array}{l}\text { Investigadora: ¿Y eso te dice o muestra algo? } \\
\text { Profesora: ¡Demuestra que estoy en lo cor- } \\
\text { recto! Porque no hubo cambios. Si el prome- } \\
\text { dio hubiera oscilado mucho, entonces sería } \\
\text { preocupante. Pero, se mantuvo constante a } \\
\text { la medida que fuimos tomando más infor- } \\
\text { mación. }\end{array}$ \\
$\begin{array}{l}\text { Investigadora: ¿Entonces eso ayudó? } \\
\text { Profesora: Sí! }\end{array}$ & \\
\hline
\end{tabular}

El uso de la herramienta identificación de la media de TinkerPlots podría haber sido una estrategia interesante de retroalimentación visual que se podría explorar si las variaciones de los promedios en función del tamaño de las muestras son registradas por la investigadora y confrontadas en cada nivel de confianza establecido. El registro visual de la variación del promedio en una tabla o por medio de otros recursos del TinkerPlots, podría servir como un soporte a la profesora para identificar los progresos del promedio y su propio progreso en la comprensión y significancia media de los datos. De esta manera, la retroalimentación dirigida contribuiría a la profesora a ampliar su razonamiento sobre los datos, ya que, con esa estrategia, la poca variación de la media podría haber sido sistemáticamente percibida.

Como una manera de finalizar la tarea, la investigadora optó por presentar la población de peces a la profesora y solicitó que confirmara si el análisis que ella desarrolló a lo largo de la tarea fue adecuado a la tendencia encontrada en la población, conforme al siguiente fragmento de diálogo:

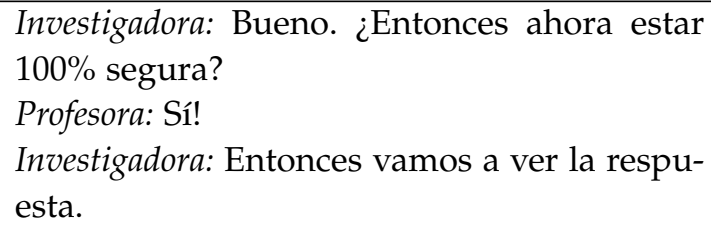




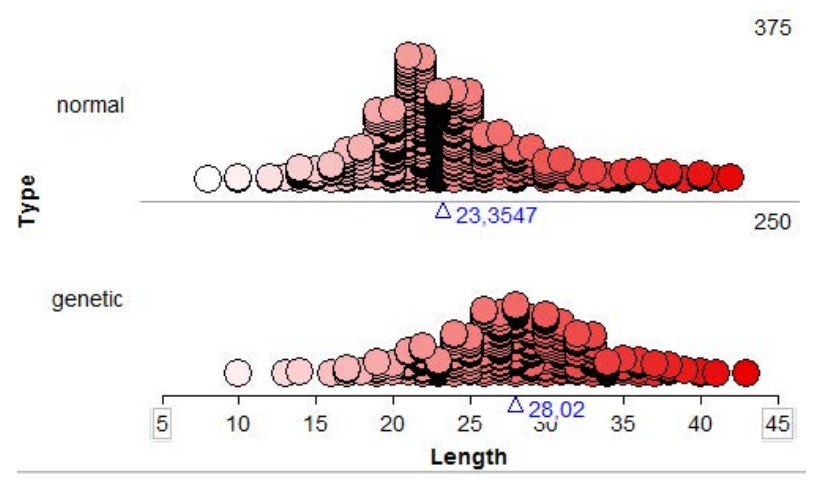

Figura 1.5: Pantalla de TinkerPlots 2.0 utilizada en la intervención con la profesora y la población de 625 peces.

Investigadora: Entonces, aquí tenemos los 625 casos. Y la longitud promedio de cada grupo. ¿Y ahí, su hipótesis se confirmó? ¿Se valida en esos datos?

Profesora: Yo creo que sí. Ahora estoy segura que sí.

Investigadora: ¿Por qué crees que tomamos muestras en lugar de estudiar todos los casos que teníamos aquí? (...) Pero, ¿por qué entonces no tomamos todo en una situación real?

Profesora: ¡Es que tiene que contar todo, no es asíl? Y la muestra es la parte significativa, ¿no? Es lo que se tiene en común entre los grupos que se extraen.

Investigadora: ¡Sí! Entonces usted puede estudiar todos los casos a través de algunos de ellos, es así? ¿En qué situaciones?

Profesora: Siempre y cuando sea un grupo significativo a la investigación. ¿Me explico? Digamos, vamos a hacer una investigación sobre combustible. ¿Quiénes serían las personas interesadas? La gente que tiene coche, me explico? ¿Buscas un peatón que no consume combustible? ¡No! Entonces, ese grupo es favorable a la investigación. Su muestra debe formase con ese grupo.
La profesora presenta los datos de la población y alienta a la docente a confrontar sus análisis y el resultado final.

En esta situación final de la realización de la tarea, la profesora solicita más explícitamente una retroalimentación de la investigadora sobre sus conclusiones.

La investigadora ofrece una retroalimentación corta para las preguntas planteadas por la profesora al mismo tiempo que plantea una cuestión sobre situaciones de utilización de los aprendizajes sobre muestreo. La profesora demuestra comprender el contexto de uso de las muestras. 
$\mathrm{Al}$ presentar toda la población a la profesora, la investigadora brinda la posibilidad de comparar las conclusiones realizadas sobre las muestras con la tendencia de todos los casos. Esto pudo ayudar a la profesora a darse cuenta que sus análisis concordaban con la tendencia real de los casos, osea, llevándola a percibir que sus muestras representaban bien la variabilidad encontrada en la población.

En el análisis de esta situación de aprendizaje mediada por el TinkerPlots, consideramos el proceso de comprensión del concepto de muestra por parte de la profesora. Se evaluó esta como una situación potencial para la formación de profesores aunque se haya desarrollado en un contexto no convencional. Incluso con estas condiciones fue posible explotar estrategias de retroalimentación, que también fue un contexto de aprendizaje.

\subsection{Consideraciones finales}

En los diálogos presentados tenemos una realidad que muestra la eficacia de la retroalimentación del profesor y supera una limitación de su operacionalización. El esfuerzo que se compartió con el lector, que se dedicó a analizar esta discusión, es el de percibir que, en situaciones de enseñanza con el uso de software, el profesor puede adquirir un enfoque instruccional a fin de que las informaciones para el desarrollo de una tarea en el ordenador sean suficientemente claras y demostradas por medio de manipulación. En estas situaciones, la retroalimentación también puede surgir como una forma de garantizar que el desempeño del aprendiz mejore cuando manipula una herramienta. Las estrategias de retroalimentación presentadas en este capítulo tuvieron en su mayoría esa función.

Sin embargo, para que este proceso pueda garantizar verdaderos aprendizajes a los alumnos, es ideal que el profesor dirija sus comentarios para la construcción de un conocimiento vinculado a las herramientas tecnológicas en cuestión. De otro modo, los enfoques instruccionales no deben limitarse a especificar las funciones de las herramientas, sino a la exploración de las mismas para que, frente a nuevas situaciones de uso, los aprendices puedan identificar cuáles son los recursos más adecuados para realizar una tarea concreta acorde al conocimiento que le está asociado, además de entender el por qué de la decisión que tomaron.

La defensa de que un conocimiento vinculado a las herramientas tecnológicas debe ser alcanzado por los sujetos ha sido contemplado en diferentes estudios (por ejemplo, Rabardel \& Waern [25], 2003). Estos autores reconocen que la inclusión de las personas en actividades que utilizan una herramienta tecnológica no es suficiente para garantizar una interacción completa entre el sujeto, la máquina y el objeto del conocimiento. Antes de eso, es importante considerar en esas actividades los procesos por los cuales las personas transforman la herramienta tecnológica en instrumento de apropiación del conocimiento. En el marco de estas tareas, la retroalimentación del profesor puede ser un elemento importante.

Como hemos enfatizamos, nuestra propuesta era iniciar una discusión sobre el uso de estrategias y contenidos de retroalimentación que podrían ser eficaces en los procesos de enseñanza y aprendizaje mediados por el uso de recursos tecnológicos. Para ello, en investigaciones futuras se debe investigar

Retroalimentación en lecciones de Estatística con Recursos Tecnológicos. C. Carvalho et al

Derechos Reservados @ 2017 Revista digital Matemática, Educación e Internet (http://tecdigital.tec.ac.cr/revistamatematica/) 
la retroalimentación en situaciones reales en lecciones de Estadística, así como en simulaciones en la formación inicial de profesores que van a enseñar esa disciplina y que pueden no haber sido instruidos en esas prácticas durante la formación inicial.

Dado que hay tantos factores que se escapan del control del profesor, pero que concurren a los procesos de enseñanza y aprendizaje, la retroalimentación se constituye en una de las pocas herramientas que los profesores pueden utilizar de manera autónoma y que está bajo su control, porque es él quien decide qué contenido o estrategia de retroalimentación utiliza en una determinada situación durante su práctica. Así, en comparación con los aspectos escolares que son impuestos por las realidades complejas, tales como: condiciones sociales de los alumnos, los contenidos curriculares predeterminados y las diversas cuestiones de gestión y organización del tiempo escolar, se puede afirmar que la retroalimentación se presenta como un elemento que pueden ser utilizado por los profesores como protagonistas y facilitadores de la enseñanza y aprendizaje de los alumnos.

\section{Bibliografía}

[1] J. Ainley \& C. Monteiro (2008). Comparing curricular approaches for statistics in primary school in England and Brasil: a focus on graphing. En C. Batanero, G. Burrill, C. Reading \& A. Rossman (Eds.), Proceedings of the Joint ICMI /IASE Study Teaching Statistics in School Mathematics: Challenges for Teaching and Teacher Education. (pp. 1-6). México: Monterrey. Recuperado de http://www.ugr.es/ ${ }^{\sim}$ icmi/iase_study/

[2] D. Ben-Zvi (2000). Toward understanding the role of technology tools in statistical learning. Mathematical Thinking and Learning, 21(1\&2), 127-155.

[3] D. Ben-Zvi, A. Bakker, \& K. Makar (2015). Learning to reason from samples. Educational Studies in Mathematics, 88(3), 291-303.

[4] D. Ben-Zvi, \& J. Garfield (Eds.) (2004). The challenge of developing statistical literacy, reasoning, and thinking. Dordrecht, The Netherlands: Kluwer Academic Publishers.

[5] L. Bergh, A. Ros \& D. Beijaard (2013). Teacher feedback during active learning: Current practices in primary schools. British Journal of educational Psychology, 83, 341-362.

[6] P. Black, \& D. Wiliam (1998). Inside the black box: Raising standards through classroom assessment. London: School of Education, King's College.

[7] S. Brookhart (2008). How to give effective feedback to your students. Alexandria, VA: Association for Supervision and Curriculum Development.

[8] C. R. Campos, O. R. Jacobini, M. L Wodewotzki; Ferreira, D.H L (2011). Educação Estatística no contexto da Educação Crítica. Bolema. Boletim de Educação Matemática (UNESP-Rio Claro), 24(39) p. 473-494.

[9] J. Fonseca, C. Carvalho, J. Conboy, M.O. Valente, A.P. Gama, E. Fiúza, \& H. Salena (2015). Changing Teachers' Feedback Practices: A Workshop Challenge. Australian Journal of Teacher Education, 40(8). 
[10] GAISE Report (2005). Guidelines for Assessment and Instruction in Statistics Education (GAISE) Report, A Pre-k-12 curriculum framework, Alexandria, VA: August 2005 -American Statistical Association. (Acesso em 30 de outubro de 2016) http://it.stlawu.edu/ ${ }^{\text {rlock/gaise/ }}$

[11] J. Garfield, \& D. Ben-Zvi (2010). Developing Students' Statistical Reasoning: Connecting Research and Teaching Practice. Dordrecht, The Netherlands: Springer.

[12] S. Grieshaber (2010). Beyond discovery: a case study of teacher interaction, young children and computer tasks. Cambridge Journal of Education, 40(1), 69-85.

[13] J. Hattie (2009). Visible learning: A synthesis of over 800 meta-analyses relating to achievement. New York: Routledge.

[14] A. Henriques, \& P. Antunes (2014). A exploração da covariação estatística por alunos do $10 .^{\circ}$ ano com o TinkerPlots. Quadrante, 23(2), 95-122.

[15] F. Jolliffe (2007). The changing brave new world of statistics assessment. In Phillips B. and Weldon L. (Eds.), The Proceedings of the ISI/IASE Satellite on Assessing 91 Student Learning in Statistics. Voorburg: International Statistical Institute, The Netherlands, CD-ROM.

[16] C. Konold, \& C.D. Miller (2005). TinkerPlots: Dynamic Data Explorations [software, Version1.0]. Emeryville, CA: Key Curriculum Press.

[17] C. E. Lopes (2012). A Educação Estocástica na Infância. Revista Eletrônica de Educação (São Carlos), 6, 160-174.

[18] M.N.P Martins (2014). Análise das concepções de professores sobre amostragem com o uso do software TinkerPlots 2.0. (Dissertação de Mestrado). Universidade Federal de Pernambuco, Brasil.

[19] C.E.F. Monteiro, L.M.T.L. Carvalho, \& J.M. Ainley (2013). O TinkerPlots como recurso para o ensino e a aprendizagem de conteúdos de Estatística no Ensino Fundamental. In R. Borba \& C. Monteiro (Eds.), Processos de Ensino e Aprendizagem em Educação Matemática (pp. 133-166). Recife, PE: Universitária UFPE.

[20] C.E.F. Monteiro, \& M.N.P. Martins (2016). Possibilidades de recursos para o ensino de probabilidade nos anos iniciais. Em Teia, 7(1) pp, 1-5.

[21] C. E. F. Monteiro ; C. F. Carvalho ; M. N. Martins (2015). O feedback em situações de aprendizagem mediadas por recursos tecnológicos. In: Carvalho, C.; Conboy, J.. (Org.). O feedback em situações de aprendizagem mediadas por recursos tecnológicos. 1ed. (pp. 377-414) Lisboa: Instituto de Educação da Universidade de Lisboa.

[22] P. Orsmond, S. Merry, \& K. Reiling (2005). Biology students? utilization of tutors' formative feedback: A qualitative interview study. Assessment and Evaluation in Higher Education, 30, 369-386.

[23] D. Pratt, N. Davies \& D. Conner (2011). The Role of Technology in Teaching and Learning Statistics. In: In C. Batanero, G. Burril, \& C. Reading, (Eds), Teaching statistics in school mathematics - challenges 
for teaching and teacher education (pp.97-107). Dordrecht: Springer.

[24] S. Quintas, R. Tomás Ferreira, \& H. Oliveira (2013). O conhecimento didático do professor no ensino da variação estatística. En J. M. Contreras, G.R., Cañadas, M. M. Gea \& P. Arteaga (Eds.), Actas de las Jornadas Virtuales en Didáctica de la Estadística, Probabilidad y Combinatoria: Vol.1. (pp. 439-446). Granada: Universidad de Granada.

[25] P. Rabardel, \& Y. Waern (2003). From artefact to instrument. Interacting with Computers, 15 (5), 641-645.

[26] A. Rubin, B. Bruce, \& Y. Tenney (1991). Learning about sampling: Trouble at the core of statistics. En D. Vere-Jones (Ed.), Proceedings of the Third International Conference on Teaching Statistics. Vol. 1 (pp. 314-319). Voorburg: International Statistical Institute.

[27] D.R. Sadler (1989). Formative assessment and the design of instructional systems. Instructional Science, 18, 119-144.

[28] M.R. Weaver (2006). Do students value feedback? Student perceptions of tutors' written responses. Assessment and Evaluation in Higher Education, 31, 379-394.

[29] G. Wiggins (2012). Seven keys to effective feedback. Feedback for learning, 70(1), 10-16. 